\title{
Fertility Care Provided by a Public Health Hospital to Viral-Infected Couples: A Case-Control Study
}

\author{
Sofia Figueiredo, Sónia Correia, Graça Pinto, Teresinha Simões* \\ Maternidade Dr. Alfredo da Costa, Nova Medical School, Lisbon, Portugal \\ Email: ^hteresinhasimoes@gmail.com
}

How to cite this paper: Figueiredo, S., Correia, S., Pinto, G. and Simões, T. (2021) Fertility Care Provided by a Public Health Hospital to Viral-Infected Couples: A Case-Control Study. Open Journal of $\mathrm{Ob}$ stetrics and Gynecology, 11, 1013-1025. https://doi.org/10.4236/ojog.2021.118095

Received: July 11, 2021

Accepted: August 14, 2021

Published: August 17, 2021

Copyright $\odot 2021$ by author(s) and Scientific Research Publishing Inc. This work is licensed under the Creative Commons Attribution International License (CC BY 4.0).

http://creativecommons.org/licenses/by/4.0/

\section{(c) (i) Open Access}

\begin{abstract}
Objective: To evaluate the results of ART (clinical pregnancies and baby home rates) in couples infected with HBV, HCV, HIV or mixed in a Referral center. Patients: The study is a retrospective case-control study comprising 1587 cycles/1064 couples in the control group and 237 cycles/164 couples in the infected group, submitted to in vitro fertilization, intracytoplasmic sperm injection and embryo transfer. Two branches from the infected group were created: the HIV subgroup, comprising couples with one or both partners infected with HIV-1 (84 cycles/67 couples), and the HCV subgroup, including at least one partner infected with HCV ( 45 cycles/29 couples). Results: The infected group presented higher infertility duration $(P<0.001)$ and so did the HCV subgroup $(P<0.001)$. Tubal infertility was increased in the infected group $(P<0.001)$, and in the HIV $(P<0.001)$ and HCV $(P=0.01)$ subgroups. Oocytes and oocytes in metaphase II numbers were lower in the infected group, but not in the HIV and HCV subgroups. Clinical pregnancy rate was lower in the infected group (25.74\%) when compared to the control (34.66\%), probably due to the contribution of HCV individuals (17.78\%), but not of HIV (28.57\%). Baby home rate was lower in the infected group (21.52\%) relative to the control group (28.42\%), but no differences were found in the HIV (27.38\%) and HCV (15.56\%) subgroups. Conclusions: Despite the low clinical pregnancy rate in the HCV subgroup, baby home rates did not change from control. These data seem promising for couples with viral infections who wish to conceive.
\end{abstract}

\section{Keywords}

Viral Infections (HIV, HBV and HCV), ART

\section{Introduction}

The United Nations AIDS Report [1] estimated that around 37 million people 
are infected with the human immunodeficiency virus (HIV) worldwide and around 17 million people undergo highly active antiretroviral treatment (HAART). The World Health Organization report [2] predicted that about 80 million people live with chronic hepatitis C (HCV). Furthermore, about 257 million people were estimated to be living with hepatitis B (HBV) in 2015 [3].

The advance of antiretroviral therapies has allowed seropositive people to live with higher expectancy and better quality of life, and adequate treatment allows infected couples to conceive without the risk of infecting the offspring [4] [5] [6]. Nowadays, almost no horizontal or vertical infection occurs in couples submitted to assisted reproductive technologies (ART) [6] [7] [8] [9]. An interesting study performed by Del Romero and collaborators [10] reported that in the absence of fertility problems, it is possible for serodiscordant couples to conceive naturally under controlled conditions in terms of safety and effective reproduction. The same authors showed that $66 \%$ of couples achieved at least one pregnancy [10]. However, most couples face issues other than the risk of infection and these include infertility which resulted from the infection, regardless of whether couples are serodiscordant or seroconcordant [11] [12] [13]. The data available so far are very disperse, but some studies state that couples infected with HIV present higher infertility rates and duration [13] [14]. Indeed, it was found that women infected with HIV presented lower number of retrieved oocytes [14]. Another case-control study reported that women infected with HCV presented a poorer ovarian response as they need more follicle-stimulating hormone (FSH) administration to obtain an equal number of oocytes [15]. Couples infected with HCV show a significant decrease of the pregnancy rate even after administration of higher doses of gonadotrophins, without changing the duration of stimulation [12]. Also, Terriou and collaborators [16] observed that equal pregnancy rates in HIV-positive women were achieved when a longer duration of stimulation was applied. An interesting finding by Coll and colleagues [17] showed that the rate of pregnancy was higher in HIV-positive women when oocytes were donated by healthy women. These HIV-positive women faced ovarian resistance to hyperstimulation because higher doses of gonadotrophins were needed to stimulate them adequately [17].

Overall, the above-mentioned data reported that the difficulties to conceive may reside in the aggravation of infertility, and adequate gonadotrophin stimulation as well as the duration of treatment should be taken into consideration.

In view of the evidence thus established, we aim at assessing the results of IVF cycles of our group infected with HBV, HCV and HIV-1 compared with a control group of uninfected couples.

\section{Material and Methods}

\subsection{Patients and Inclusion Criteria}

This study is a retrospective and case-control study with data collected from February 2013 to May 2019. Couples attended the Reproductive Department of 
the Maternity Dr. Alfredo da Costa, Centro Hospitalar Universitário Lisboa Central, Lisbon, Portugal, for assisted reproduction. Our hospital is the only public unit for couples with viral infections and serves as a referral center in Portugal.

Two main groups were created in this study: the control group, which comprises uninfected couples who searched for assisted reproduction to conceive, and the infected group that includes couples in which at least one partner is infected with HIV-1, HIV-2, HCV and HBV. Two branches from the infected group were created: the HIV subgroup, which comprises couples with one (17 women and 39 men) or both partners infected with HIV-1 (28 couples), and the HCV subgroup, which includes at least one partner infected with HCV (12 women and 33 men). The study comprised 1587 cycles/1064 couples in the control group, 237 cycles/164 couples in the infected group, 84 cycles/67 couples in the HIV subgroup, and 45 cycles/29 couples in the HCV subgroup.

$\mathrm{HIV}$ and HCV individuals were included in this study if the detection by reverse polymerase chain reaction (PCR) was positive. Viruses' antibodies were detected in serum using enzyme-linked immunosorbent assay (ELISA) or Western blot. For HIV-1-positive women, CD4 cell counts should be higher than 200 cells $/ \mathrm{mm}^{3}$ and the plasma HIV-RNA of women who are under HAART must be below the detection limit before ART. Patients were followed until the pregnancy test by our center and, if positive, the results of the pregnancy were collected at the end of the gestation.

The following parameters were analyzed in all groups and subgroups: tobacco use, duration of infertility, progeny prior to treatment and types of infertility (tubal, male, and mixed).

Patients were considered suitable for assisted reproduction treatment if their clinical, immunological, and virological criteria were compatible for pregnancy outcome, and the risk of vertical transmission was minimal.

\subsection{Procedures}

Both partners were evaluated. The antral follicles count and the antimüllerian hormone values $(\mathrm{AMH})$ were determined before stimulation. ART included the techniques of in vitro fertilization (IVF) or intracytoplasmic sperm injection (ICSI) and, in some cases, testicular epididymal sperm extraction was performed. Total doses of gonadotrophins included the recombinant FSH and either human menopausal gonadotrophins (hMG) or luteinizing hormone (LH). Ovarian stimulation started on day 3 of the menstrual cycle and levels of FSH and estradiol $\left(E_{2}\right)$ were assessed. The stimulation protocol was adjusted in order to achieve the best ovarian response. Follicle development was examined by ultrasound and by plasma $\mathrm{E}_{2}$ levels. Ovulation was triggered with human chorionic gonadotrophin (hCG) when at least 3 follicles had reached $17 \mathrm{~mm}$ and oocyte retrieval was performed 35 - 37 hours after triggering ovulation. The number of oocytes and oocytes in metaphase II, and the number of embryos were determined. 
Clinical pregnancy was determined by transvaginal ultrasound through positive fetal heart pulsation at 6 weeks of gestational age.

\subsection{Statistical Analysis}

Statistical significance was assessed comparing the total infected group with the control group. Each branch of the infected group, HIV and HCV subgroups, was also individually compared with the control group. Mann-Whitney post-hoc test or Fisher's exact test was used as indicated in the table's notes and figure legends. Data are expressed as mean values \pm standard deviation, proportion in percentage \pm average amplitude of the $95 \%$ confidence interval, or proportion in percentage. Data were considered statistically significant when $P<0.05$. All statistical analyses were performed using IBM $^{\circledR}$ SPSS $^{\circledR}$ v24.

Data were collected anonymously, and the study was approved by the local Institutional Review Board.

\section{Results}

\subsection{Sample Group and Sociodemographic Characterization of the Infected Group}

The average ages of both female and male individuals were similar despite the statistical significance observed in the infected group when compared to control. Female body mass index (BMI) differences were statistically significant between the infected group and the control. We believe that this difference is not relevant in our study. The smoking status was not different in the infected group (Table 1).

Infertility was assessed in both groups by determining its duration as well as its etiology. Our results stated that duration of infertility was augmented in the infected group. No differences were observed in the average progeny measured in both groups. However, tubal-related infertility was significantly higher in the infected group whereas the male-related infertility was significantly lower (Table $1)$.

No differences were observed in the numbers of antral follicles, or on the average of FSH and antimüllerian hormone (AMH) in the infected and control groups (Table 1).

\subsection{Cycle Parameters of the Infected Group}

The amount of total gonadotrophins administered was similar when we compared the infected group with the control. Similarly, the duration of ovarian stimulation, $\mathrm{E}_{2}$ levels at the hCG administration day, and pregnancy rate/transfer did not show statistically significant differences. However, absolute numbers of oocytes, oocytes in metaphase II and embryo counts were significantly lower in the infected group (Table 1).

\subsection{Clinical Pregnancy and Baby Home Rates in the Infected Group}

Clinical pregnancy rate was found to be significantly lower in the infected group 
Table 1. Demographic characteristics and cycle results of control and viral infected population.

\begin{tabular}{|c|c|c|c|}
\hline Criteria & Control & Infected group & $P$ value \\
\hline Number of cycles & 1587 & 237 & \\
\hline Age F (years) & $34.42 \pm 3.82$ & $35.27 \pm 3.32$ & 0.0007 \\
\hline Age $M$ (years) & $36.56 \pm 5.05$ & $38.57 \pm 5.19$ & $<0.001$ \\
\hline BMI F $\left(\mathrm{kg} / \mathrm{m}^{2}\right)$ & $23.92 \pm 3.60$ & $24.88 \pm 3.91$ & 0.0006 \\
\hline BMI M $\left(\mathrm{kg} / \mathrm{m}^{2}\right)$ & $26.60 \pm 7.75$ & $26.19 \pm 3.47$ & 0.62 \\
\hline Tobacco F & $1.68 \pm 4.09$ & $2.14 \pm 4.87$ & 0.64 \\
\hline Tobacco M & $4.06 \pm 7.28$ & $5.11 \pm 8.40$ & 0.14 \\
\hline Infertility (years) & $5.19 \pm 2.54$ & $6.05 \pm 3.43$ & 0.0001 \\
\hline With progeny & $0.07 \pm 0.29$ & $0.09 \pm 0.33$ & 0.14 \\
\hline Tubal (\%) & 7.50 & 20.68 & $<0.001$ \\
\hline Male (\%) & 46.12 & 29.54 & $<0.001$ \\
\hline Mixed (\%) & 27.35 & 25.74 & 0.61 \\
\hline Antral follicle counts & $14.73 \pm 7.94$ & $13.81 \pm 7.05$ & 0.11 \\
\hline FSH (IU/mL) & $7.00 \pm 4.45$ & $7.02 \pm 2.33$ & 0.40 \\
\hline $\mathrm{AMH}(\mathrm{ng} / \mathrm{mL})$ & $2.09 \pm 2.21$ & $1.93 \pm 1.82$ & 0.41 \\
\hline Total dose of gonadotrophins (IU) & $2267.10 \pm 1273.92$ & $2257.47 \pm 1263.52$ & 0.85 \\
\hline Stimulation (days) & $10.06 \pm 1.99$ & $9.96 \pm 2.26$ & 0.29 \\
\hline $\mathrm{E}_{2}(\mathrm{pg} / \mathrm{mL})$ & $1490.00 \pm 765.88$ & $1482.48 \pm 887.32$ & 0.38 \\
\hline Oocytes count & $7.69 \pm 4.56$ & $7.16 \pm 5.19$ & 0.03 \\
\hline Metaphase II & $6.22 \pm 3.87$ & $5.78 \pm 4.43$ & 0.02 \\
\hline Embryo counts & $3.98 \pm 2.85$ & $3.57 \pm 3.18$ & 0.002 \\
\hline Pregnancy rate/transfer (\%) & 36.17 & 30.93 & 0.15 \\
\hline
\end{tabular}

Note: Groups are represented as: Control (uninfected population), $\mathrm{n}=1587$; and Infected population (at least one partner infected with HIV, HBV and HCV), $n=237$. AMH: Antimüllerian hormone; BMI: Body mass index; $\mathrm{E}_{2}$ : Estradiol levels at the hCG administration day; F: Female individuals; FSH: Follicle-stimulating hormone; M: Male individuals. Data are represented as mean values \pm standard deviation using Mann-Whitney post-hoc test or as proportion in percentage using Fisher's exact test. Statistical significance was set for $P<0.05$.

$(25.74 \% \pm 5.57 \% ; 95 \% \mathrm{CI}: 20.17-31.30 ; P=0.006)$ when compared to the control group (34.66\% $\pm 2.34 \%$; 95\% CI: 32.32 - 37.00) (Figure 1(A)). Baby home rate was also altered in the infected group, showing lower values $(21.52 \% \pm$ 5.23\%; 95\% CI: 16.29 - 26.75; $P=0.006)$ than control $(28.42 \% \pm 2.22 \%$; $95 \% \mathrm{CI}$ : 26.20 - 30.64) (Figure 1(B)).

\subsection{Sample Group and Sociodemographic Characterization of HIV- and HCV-Infected Subgroups}

After establishing IVF cycle results, clinical pregnancy, and baby home rates in the infected group population, we also aim at determining whether these parameters 


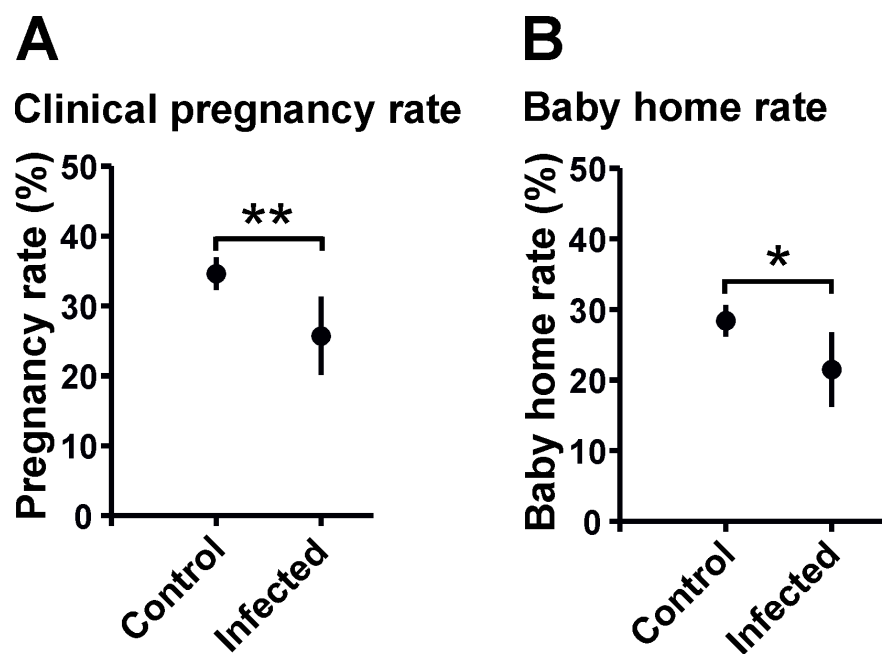

Figure 1. Clinical pregnancy and baby home rates of infected group population. Clinical pregnancy (A) and baby home rates (B) were obtained from control (uninfected individuals; $\mathrm{n}=1587$ ) and infected group (at least one partner infected with HIV, HCV and $\mathrm{HBV} ; \mathrm{n}=237$ ). Data are represented as proportion in percentage \pm average amplitude of the $95 \%$ confidence interval and statistical significance were set for $P<0.05$ using Fisher's exact test.

were altered in two subgroups belonging to this group, the HIV and HCV. Here, each subgroup was compared to controls.

Female and male individuals from HCV subgroup were older than the respective controls. BMI was not different among groups. Again, we believe that these differences will not interfere with our analysis. Smoking status was increased in all individuals belonging to the HCV subgroup (Table 2).

The duration of infertility was significantly higher only in the HCV subgroup, but the average progeny of both HIV and HCV was not different from control (Table 2).

Results regarding infertility etiology showed that both HIV and HCV subgroups have higher tubal-related infertility than controls. As for male-related and mixed infertilities, they were reduced only for the HIV subgroup (Table 2).

No differences were observed for antral follicles count and FSH amount for both subgroups. However, AMH was decreased only in the HIV subgroup (Table 2).

\subsection{Cycle Parameters of HIV- and HCV-Infected Subgroups}

Total doses of gonadotrophins, duration of stimulation, levels of $E_{2}$ at the hCG administration day, numbers of oocyte and oocytes in metaphase II, and embryo counts of each subgroup (HIV or HCV) did not differ from controls. On the other hand, pregnancy rate/transfer was significantly decreased in HCV subgroup (Table 2).

\subsection{Clinical Pregnancy and Baby Home Rates in HIV-and HCV-Infected Subgroups}

After observing a decrease in clinical pregnancy rate in the total infected group, 
Table 2. Demographic characteristics and cycle results of control and of the two subgroups belonging to the infected population, HIV and HCV.

\begin{tabular}{|c|c|c|c|c|c|}
\hline \multirow{3}{*}{ Criteria } & \multirow{3}{*}{$\begin{array}{l}\text { Control } \\
\text { Result }\end{array}$} & \multicolumn{4}{|c|}{ Subgroups of the infected population } \\
\hline & & \multicolumn{2}{|l|}{ HIV } & \multicolumn{2}{|l|}{$\mathrm{HCV}$} \\
\hline & & Result & $P$ value & Result & $P$ value \\
\hline Number of cycles & 1587 & 84 & & 45 & \\
\hline Age F (years) & $34.42 \pm 3.82$ & $35.15 \pm 3.29$ & 0.08 & $36.76 \pm 1.98$ & $<0.001$ \\
\hline Age $\mathbf{M}$ (years) & $36.56 \pm 5.05$ & $37.68 \pm 5.70$ & 0.16 & $39.36 \pm 4.44$ & 0.0001 \\
\hline BMI F $\left(\mathrm{kg} / \mathrm{m}^{2}\right)$ & $23.92 \pm 3.60$ & $24.65 \pm 3.84$ & 0.08 & $24.46 \pm 3.82$ & 0.45 \\
\hline BMI M $\left(\mathrm{kg} / \mathrm{m}^{2}\right)$ & $26.60 \pm 7.75$ & $25.88 \pm 3.17$ & 0.42 & $25.45 \pm 3.43$ & 0.09 \\
\hline Tobacco F & $1.68 \pm 4.09$ & $0.81 \pm 2.54$ & 0.02 & $4.15 \pm 7.09$ & 0.006 \\
\hline Tobacco $\mathbf{M}$ & $4.06 \pm 7.28$ & $3.90 \pm 8.08$ & 0.48 & $11.16 \pm 9.53$ & $<0.001$ \\
\hline Infertility (years) & $5.19 \pm 2.54$ & $5.12 \pm 3.46$ & 0.74 & $7.66 \pm 4.68$ & 0.0006 \\
\hline With progeny & $0.07 \pm 0.29$ & $0.10 \pm 0.30$ & 0.14 & $0.09 \pm 0.36$ & 0.75 \\
\hline Tubal (\%) & 7.50 & 23.81 & $<0.001$ & 17.78 & 0.01 \\
\hline Male (\%) & 46.12 & 27.38 & $<0.001$ & 33.33 & 0.09 \\
\hline Mixed (\%) & 27.35 & 10.71 & $<0.001$ & 40.00 & 0.06 \\
\hline Antral follicle counts & $14.73 \pm 7.94$ & $12.93 \pm 6.12$ & 0.07 & $13.95 \pm 7.58$ & 0.42 \\
\hline FSH (IU/mL) & $7.00 \pm 4.45$ & $7.13 \pm 2.45$ & 0.51 & $7.44 \pm 2.10$ & 0.09 \\
\hline $\mathrm{AMH}(\mathrm{ng} / \mathrm{mL})$ & $2.09 \pm 2.21$ & $1.13 \pm 1.39$ & 0.02 & $1.48 \pm 1.09$ & 0.37 \\
\hline $\begin{array}{c}\text { Total dose of } \\
\text { gonadotrophins (IU) }\end{array}$ & $2267.10 \pm 1273.92$ & $2161.02 \pm 1141.03$ & 0.98 & $2230.28 \pm 1059.97$ & 0.68 \\
\hline Stimulation (days) & $10.06 \pm 1.99$ & $9.86 \pm 2.27$ & 0.87 & $9.78 \pm 2.26$ & 0.29 \\
\hline $\mathrm{E}_{2}(\mathrm{pg} / \mathrm{mL})$ & $1490.00 \pm 765.88$ & $1500.65 \pm 1076.99$ & 0.25 & $1262.62 \pm 669.63$ & 0.06 \\
\hline Oocytes count & $7.69 \pm 4.56$ & $7.31 \pm 5.67$ & 0.18 & $7.11 \pm 4.83$ & 0.29 \\
\hline Metaphase II & $6.22 \pm 3.87$ & $6.08 \pm 5.46$ & 0.13 & $5.95 \pm 4.35$ & 0.45 \\
\hline Embryo counts & $3.98 \pm 2.85$ & $3.76 \pm 3.63$ & 0.06 & $3.82 \pm 3.65$ & 0.28 \\
\hline $\begin{array}{c}\text { Pregnancy } \\
\text { rate/transfer (\%) }\end{array}$ & 36.17 & 40.00 & 0.55 & 20.51 & 0.04 \\
\hline
\end{tabular}

Note: Groups are represented as: Control (uninfected population), $\mathrm{n}=1587$; HIV subgroup, $\mathrm{n}=84$; and $\mathrm{HCV}$ subgroup, $\mathrm{n}=45$. Data are represented as mean values \pm standard deviation using Mann-Whitney post-hoc test or as proportion in percentage using Fisher's exact test. Statistical significance was set for $P<$ 0.05 .

we ought to determine if it might be due to the contribution of HIV and/or HCV subgroups. No differences were observed between HIV $(28.57 \% \pm 9.66 \%$; $95 \%$ CI: $18.91-38.23 ; P=0.251)$ and the control $(34.66 \% \pm 2.34 \%$; $95 \%$ CI: $32.32-$ $37.00)$. On the other hand, clinical pregnancy rate was significantly reduced in HCV subgroup $(17.78 \% \pm 11.17 \%$; 95\% CI: $6.61-28.95 ; P=0.019)$ (Figure 2(A)).

Neither HIV $(27.38 \% \pm 9.54 \%$; 95\% CI: $17.84-36.92 ; P=0.843)$ nor HCV (15.56\% $\pm 10.59 \%$; 95\% CI: $4.97-26.15 ; P=0.058)$ baby home rates differed significantly from control (28.42\% $\pm 2.22 \%$; $95 \%$ CI: 26.20 - 30.64) (Figure 2(B)). 

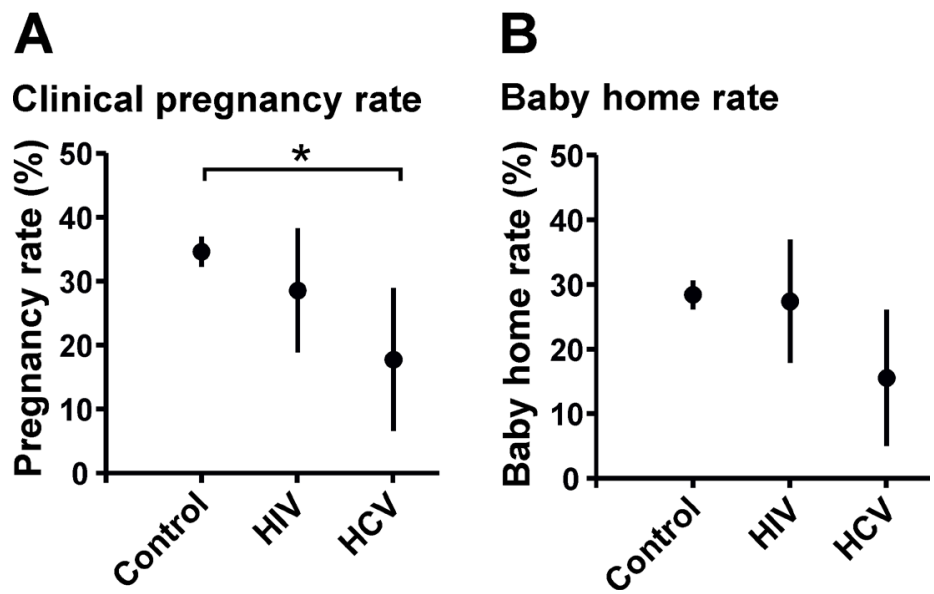

Figure 2. Clinical pregnancy and baby home rates of HIV and HCV subgroups belonging to the infected group. Clinical pregnancy (A) and baby home (B) rates were obtained from control (uninfected individuals; $\mathrm{n}=1587$ ), HIV (at least one partner infected with HIV-1; $\mathrm{n}=84$ ) and HCV (at least one partner infected with HCV; $\mathrm{n}=45$ ). Data are represented as proportion in percentage \pm average amplitude of the $95 \%$ confidence interval and statistical significance were set for $P<0.05$ using Fisher's exact test.

\section{Discussion}

In this study, we aimed at determining IVF cycle results from a Portuguese infected group which resorted to ART to conceive. We also sought to analyze $\mathrm{HIV}$ - and HCV-infected couples individually, two major subgroups of this infected group. The main findings of the present study reside in lower baby home and clinical pregnancy rates in total infected group. In particular, we verified that the clinical pregnancy rate was very low in the HCV subgroup, suggesting that HCV individuals within the infected group contributed the most to this reduction. However, the reduction in baby home rates was only statistically significant for the infected group and not for each subgroup. This could be explained by the high variability observed in both HIV and HCV subgroups when analyzed separately.

The development of HAART provides an increase in life expectancy and quality of life. Administration of specific HAART solutions makes it possible for infected couples to conceive [18] [19]. Furthermore, vertical transmission is almost inexistent when couples undergo ART [7] [8] [9], although they may face infertility-related issues caused by the diseases.

The data provided in the present work was obtained from the only Portuguese Public Health Hospital that provides free of charge fertility care to couples with viral infections.

Our findings revealed that the pregnancy rate was decreased in HCV subgroup. Pieces of evidence demonstrated that HCV serodiscordant couples face difficulties in conceiving [12] [15]. These impairments included poorer ovarian response to stimulation, which could be associated with low ovarian reserve, resulting in an increment of cancelled cycles [15]. Additionally, when there was a response to stimulation, low levels of $\mathrm{E}_{2}$ and reduced number of antral follicles 
were observed [12]. Our numbers did not differ from control regarding stimulation duration, doses of gonadotrophins administered and $\mathrm{E}_{2}$ levels. Also, we did not find any differences in the number of antral follicles, or in FSH and AMH levels. Most likely, the HCV group might have required an increased duration of stimulation to reach equivalent pregnancy rates to control. One study pointed out that HCV-infected women required more gonadotrophins doses to obtain similar peak levels of $E_{2}$, but lower numbers of embryos were obtained [15]. The present data suggest that tubal causes of infertility might be the reason behind low pregnancy rates. Another possible reason to explain low pregnancy rates could be the decreased pregnancy rate/transfer as implantation might not have occurred after embryo transfer. Hanafi and collaborators [12] found a decrease in implantation rates in women infected with HCV, but another study showed that HCV did not have any influence in this parameter [15]. We propose that the presence of the disease alongside other infertility outcomes may increase the difficulty of couples to conceive. In fact, male individuals carrying HIV, HCV or HBV present reduced semen volume, sperm count and motility, and increased abnormal sperm morphology [20] [21] [22] [23]. It has also been demonstrated that HIV and HBV can increase the prevalence of unilateral and bilateral tubal-related, and uterine infertilities [13] [16] [24]. Another parameter that could interfere with pregnancy rate is smoking habits as we observed a significant increase in tobacco use in HCV couples. In fact, women who smoke have a high prevalence of poorer ovarian reserves and lower pregnancy rates [25] and men have lower sperm count, motility, and viability [26]. These effects, in association with the disease, may aggravate the impairment to conceive.

We observed that the rate of clinical pregnancy did not significantly change in the HIV subgroup. Other studies corroborate ours, observing that HIV did not have any effect in the total dose of administered gonadotrophins, the duration of stimulation, $\mathrm{E}_{2}$ levels, or in the number of mature follicles, oocytes, and embryos. The number of clinical pregnancies in HIV-positive women also did not change [13] [27]. In a similar line of study, another group did not find any differences in AMH levels and antral follicles count between HIV-1-positive and non-infected women [28]. These authors did not note any differences in ovarian stimulation, duration, total gonadotropins units administered, except for a difference in $E_{2}$ levels, but lower pregnancy rates were observed [28], in line with the results we obtained in this work. Except for AMH levels that were reduced in HIV subgroup, no other differences were observed for the number of antral follicles and the concentrations of FSH in relation to controls, which suggests that all groups possess similar ovarian reserve. Gonadotrophin's administration and duration of stimulation did not differ in the HIV subgroup. However, the number of oocytes, the number of oocytes under metaphase II and the number of embryos were reduced in the infected group, but not specifically in the HIV subgroup. Once again, we propose that ovarian stimulation is not compromised by HIV-1. 
Baby home rates were significantly lower in total infected group, but it did not differ across the subgroups. Vankerkem and colleagues [14] did not see any differences in delivery rates in serodiscordant (HIV-positive women or HIV-positive male partners) or in seroconcordant couples. Curiously, clinical pregnancy and baby home rates in the HIV subgroup matched those of the control group. In contrast, Ohl and collaborators [29] showed that the rate of newborns was reduced in HIV- and HBV-infected women, but no differences were observed in HCV-infected women. The number of individuals in the HCV group [29] was drastically smaller than that in subgroups HIV and HBV, and it did not offer a solid estimate of the number of newborns. In this study, we also present a low number of individuals within the HIV and HCV subgroups. In 2015, the rate of HIV and HCV in Portugal were 9.5 and 1.1 per 100,000 population, respectively [30] [31], so higher numbers of infected individuals undergoing ART should not be expected. Moreover, no evidence is available regarding the incidence of infected individuals who are assisted in private health centers that could help us to establish a comparison with our data. However, of the $10 \mathrm{HCV}$-positive women with negative male partners belonging to the HCV subgroup, only one woman had a clinical pregnancy that ended in a miscarriage in the first trimester. In fact, one study showed a substantial decrease of live-birth rates in HIV-infected women with age above 35 years [32].

\section{Conclusion}

The present work reveals that the group with viral infection presented a similar response to stimulation. Despite the HCV subgroup presenting lower clinical pregnancy rate $(17.78 \%$ vs. $34.66 \%$ in the control group), the baby home rate was similar to the uninfected control group.

\section{Capsule Summary}

ART results from couples with viral infections (HBV, HCV, HIV or mixed) in a Portuguese public hospital. Despite low pregnancy rates, promising results on baby home rates were observed.

\section{Acknowledgements}

This study was supported by Gedeon Richter Portugal.

\section{Conflicts of Interest}

The authors declare no conflicts of interest regarding the publication of this paper.

\section{References}

[1] United Nations Global AIDS Update (UNAIDS). Global Report: 2016 Statistics. https://www.unaids.org/sites/default/files/media asset/global-AIDS-update-2016 e n.pdf 
[2] Global Report on Access to Hepatitis C Treatment, Focus on Overcoming Barriers. World Health Organization Report: 2016. https://apps.who.int/iris/handle/10665/250625

[3] Global Hepatitis Report. World Health Organization Report: 2017. https://www.who.int/hepatitis/publications/global-hepatitis-report2017/en

[4] Semprini, A.E., Macaluso, M., Hollander, L., Vucetich, A., Duerr, A., Mor, G., et al. (2013) Safe Conception for HIV-Discordant Couples: Insemination with Processed Semen from the HIV-Infected Partner. American Journal of Obstetrics and Gynecology, 208, 402.e1-9. https://doi.org/10.1016/j.ajog.2013.02.009

[5] Vernazza, P.L., Graf, I., Sonnenberg-Schwan, U., Geit, M. and Meurer, A. (2011) Preexposure Prophylaxis and Timed Intercourse for HIV-Discordant Couples Willing to Conceive a Child. AIDS, 25, 2005-2008. https://doi.org/10.1097/QAD.0b013e32834a36d0

[6] Mmeje, O., Cohen, C.R. and Cohan, D. (2012) Evaluating Safer Conception Options for HIV-Serodiscordant Couples (HIV-Infected Female/HIV-Uninfected Male): A Closer Look at Vaginal Insemination. Infectious Diseases in Obstetrics and Gynecology, 2012, Article ID: 587651. https://doi.org/10.1155/2012/587651

[7] Kashima, K., Takakuwa, K., Suzuki, M., Makino, M., Kaneko, S., Kato, S., et al. (2009) Studies of Assisted Reproduction Techniques (ART) for HIV-1-Discordant Couples Using Washed Sperm and the Nested PCR Method: A Comparison of the Pregnancy Rates in HIV-1-Discordant Couples and Control Couples. Japanese Journal of Infectious Diseases, 62, 173-176.

[8] Nesrine, F. and Saleh, H. (2012) Hepatitis C Virus (HCV) Status in Newborns Born to HCV Positive Women Performing Intracytoplasmic Sperm Injection. African Health Sciences, 12, 58-62.

[9] Savasi, V., Oneta, M., Parrilla, B. and Cetin, I. (2013) Should HCV Discordant Couples with a Seropositive Male Partner Be Treated with Assisted Reproduction Techniques (ART)? European Journal of Obstetrics \& Gynecology and Reproductive Biology, 167, 181-184. https://doi.org/10.1016/j.ejogrb.2012.12.012

[10] Del Romero, J., Baza, M.B., Río, I., Jerónimo, A., Vera, M., Hernando, V., et al. (2016) Natural Conception in HIV-Serodiscordant Couples with the Infected Partner in Suppressive Antiretroviral Therapy: A Prospective Cohort Study. Medicine (Baltimore), 95, e4398. https://doi.org/10.1097/MD.0000000000004398

[11] Ohl, J., Partisani, M., Wittemer, C., Lang, J.M., Viville, S. and Favre, R. (2005) Encouraging Results Despite Complexity of Multidisciplinary Care of HIV-Infected Women Using Assisted Reproduction Techniques. Human Reproduction, 20, 3136-3140. https://doi.org/10.1093/humrep/dei185

[12] Hanafi, N.F., Abo Ali, A.H. and Abo el kheir, H.F. (2011) ICSI Outcome in Women Who Have Positive PCR Result for Hepatitis C Virus. Human Reproduction, 26, 143-147. https://doi.org/10.1093/humrep/deq317

[13] Santulli, P., Gayet, V., Fauque, P., Chopin, N., Dulioust, E., Wolf, J.P., et al. (2011) HIV-Positive Patients Undertaking ART Have Longer Infertility Histories than Age-Matched Control Subjects. Fertility and Sterility, 95, 507-512.

https://doi.org/10.1016/j.fertnstert.2010.09.018

[14] Vankerkem, P., Manigart, Y., Delvigne, A., Ameye, L., Konopnicki, D., Shaw-Jackson, C., et al. (2017) In Vitro Fertilization When Men, Women, or Both Partners Are Positive for HIV: A Case-Control Study. Archives of Gynecology and Obstetrics, 295, 1493-1507. https://doi.org/10.1007/s00404-017-4374-0

[15] Englert, Y., Moens, E., Vannin, A.S., Liesnard, C., Emiliani, S., Delbaere, A., et al. 
(2007) Impaired Ovarian Stimulation during in Vitro Fertilization in Women Who Are Seropositive for Hepatitis C Virus and Seronegative for Human Immunodeficiency Virus. Fertility and Sterility, 88, 607-611. https://doi.org/10.1016/j.fertnstert.2006.11.177

[16] Terriou, P., Auquier, P., Chabert-Orsini, V., Chinchole, J.M., Cravello, L., Giorgetti, C., et al. (2005) Outcome of ICSI in HIV-1-Infected Women. Human Reproduction, 20, 2838-2843. https://doi.org/10.1093/humrep/dei119

[17] Coll, O., Suy, A., Figueras, F., Vernaeve, V., Martínez, E., Mataró, D., et al. (2006) Decreased Pregnancy Rate after In-Vitro Fertilization in HIV-Infected Women Receiving HAART. AIDS, 20, 121-123. https://doi.org/10.1097/01.aids.0000196161.25647.35

[18] McCormack, S.A. and Best, B.M. (2014) Protecting the Fetus against HIV Infection: A Systematic Review of Placental Transfer of Antiretrovirals. Clinical Pharmacokinetics, 53, 989-1004. https://doi.org/10.1007/s40262-014-0185-7

[19] Spera, A.M., Eldin, T.K., Tosone, G. and Orlando, R. (2016) Antiviral Therapy for Hepatitis C: Has Anything Changed for Pregnant/Lactating Women? World Journal of Hepatology, 8, 557-565. https://doi.org/10.4254/wjh.v8.i12.557

[20] Nicopoullos, J.D., Almeida, P.A., Ramsay, J.W. and Gilling-Smith, C. (2004) The Effect of Human Immunodeficiency Virus on Sperm Parameters and the Outcome of Intrauterine Insemination Following Sperm Washing. Human Reproduction, 19, 2289-2297. https://doi.org/10.1093/humrep/deh426

[21] Lorusso, F., Palmisano, M., Chironna, M., Vacca, M., Masciandaro, P., Bassi, E., et al. (2010) Impact of Chronic Viral Diseases on Semen Parameters. Andrologia, 42, 121-126. https://doi.org/10.1111/j.1439-0272.2009.00970.x

[22] Hofny, E.R., Ali, M.E., Taha, E.A., Nafeh, H.M., Sayed, D.S., Abdel-Azeem, H.G., et al. (2011) Semen and Hormonal Parameters in Men with Chronic Hepatitis C Infection. Fertility and Sterility, 95, 2557-2559. https://doi.org/10.1016/j.fertnstert.2011.05.014

[23] La Vignera, S., Condorelli, R.A., Vicari, E., D’Agata, R. and Calogero, A.E. (2012) Sperm DNA Damage in Patients with Chronic Viral C Hepatitis. European Journal of Internal Medicine, 23, 19-24. https://doi.org/10.1016/j.ejim.2011.08.011

[24] Lao, T.T., Mak, J.S.M. and Li, T.C. (2017) Hepatitis B Virus Infection Status and Infertility Causes in Couples Seeking Fertility Treatment-Indicator of Impaired Immune Response? American Journal of Reproductive Immunology, 77, e12636. https://doi.org/10.1111/aji.12636

[25] Freour, T., Masson, D., Mirallie, S., Jean, M., Bach, K., Dejoie, T., et al. (2008) Active Smoking Compromises IVF Outcome and Affects Ovarian Reserve. Reproductive BioMedicine Online, 16, 96-102. https://doi.org/10.1016/S1472-6483(10)60561-5

[26] Mostafa, R.M., Nasrallah, Y.S., Hassan, M.M., Farrag, A.F., Majzoub, A. and Agarwal, A. (2017) The Effect of Cigarette Smoking on Human Seminal Parameters, Sperm Chromatin Structure and Condensation. Andrologia, 50, e12910. https://doi.org/10.1111/and.12910

[27] Martinet, V., Manigart, Y., Rozenberg, S., Becker, B., Gerard, M. and Delvigne, A. (2006) Ovarian Response to Stimulation of HIV-Positive Patients during IVF Treatment: A Matched, Controlled Study. Human Reproduction, 21, 1212-1217. https://doi.org/10.1093/humrep/dei493

[28] Stora, C., Epelboin, S., Devouche, E., Matheron, S., Epelboin, L., Yazbeck, C., et al. (2016) Women Infected with Human Immunodeficiency Virus Type 1 Have Poorer 
Assisted Reproduction Outcomes: A Case-Control Study. Fertility and Sterility, 105, 1193-1201. https://doi.org/10.1016/j.fertnstert.2015.12.138

[29] Ohl, J. and Partisani, M. (2007) The Desire to Become a Parent When Infected with Human Immunodeficiency Virus, Hepatitis C Virus or Hepatitis B Virus. Gynécologie Obstétrique \& Fertilité, 35, 1035-1038.

https://doi.org/10.1016/j.gyobfe.2007.06.013

[30] Surveillance Report, Annual Epidemiological Report for 2015-HIV and AIDS. https://ecdc.europa.eu/sites/portal/files/documents/AER for 2015-HIV-AIDS.pdf

[31] Surveillance Report, Annual Epidemiological Report for 2015-Hepatitis C. https://ecdc.europa.eu/en/hepatitis-c/surveillance-and-disease-data

[32] Nurudeen, S.K., Grossman, L.C., Bourne, L., Guarnaccia, M.M., Sauer, M.V. and Douglas, N.C. (2013) Reproductive Outcomes of HIV Seropositive Women Treated by Assisted Reproduction. Journal of Women's Health (Larchmt), 22, 243-249. https://doi.org/10.1089/jwh.2012.3855 